\title{
Derivatives, Products, and Pullbacks in Forman's Combinatorial Differential Forms
}

\author{
Vu Quang Huynh ${ }^{1}$, Thach Phu Nguyen ${ }^{1}$, Phuong Van Phan ${ }^{1}$ \\ ${ }^{1}$ Faculty of Mathematics and Computer Science, \\ VNU-HCM University of Science, 227 Nguyen Van Cu, District 5, Ho Chi Minh City, Vietnam \\ Correspondence to: Vu Quang Huynh, Email: hqvu@hcmus.edu.vn
}

\begin{abstract}
We study derivatives, closedness, and exactness of 0-forms and 1-forms in the theory of combinatorial differential forms constructed by Robin Forman. We give an example of a closed but not exact 1-form on a non-simply connected domain. We give a sufficient condition on the domain for a closed 1-form to be exact. We show that the product of forms proposed by Forman is not anti-commutative. We propose a definition of pullbacks of forms and show that this operation has several properties analogous to pullbacks on smooth forms.
\end{abstract}

Keywords: Combinatorial Differential Form, Exactness, Product, Anti-Commutativity, Pullback DOI: https://doi.org/10.3126/jnms.v3i1.32997

\section{Introduction}

In differential topology, the theory of smooth differential forms serves a rigorous and systematic way to operate on differentials and to do integrations on general domains. A major result is that the de Rham cohomology constructed from differential forms is isomorphic to the singular cohomology, relating differential forms to the topology of the domain, see e.g. 2, 7]. Robin Forman in the paper [5, p. 340346] introduced an analogous theory of combinatorial differential forms on cell complexes, giving a result analogous to the smooth de Rham theorem. This theory is a part of a more extensive study on combinatorial differential topology, which has achieved results mirroring deep results in smooth topology, and has found applications, see e.g. 4, 6. More recently there have been other efforts at developing combinatorial theories of differential forms, such as by the groups of J. Marsden and A. Hirani [3], E. Mansfield [8, from various perspectives, indicating growing interests.

In this article we study several properties of Forman's combinatorial differential forms for 0-forms and 1-forms. In Section 2 we discuss two ways to present 0-forms and 1-forms, as maps on chain complexes and as real functions. In Section 3 we discuss derivatives of 0 -forms and 1-forms. Section 3.3 contains an example of a closed but not exact 1-form on a non-simply connected domain. Theorem 2 gives a sufficient condition on the domain for a closed 1-form to be exact, indicating that derivatives of combinatorial forms behave similarly to exterior derivatives of smooth forms. In Section 4.1 we show that the product of forms proposed by Forman does not satisfy anti-commutativity, in contrast to the smooth case. In Section 4.2 we propose a pullback operation on forms and prove linearity and naturality of this pullback.

\section{Combinatorial Forms and Representations of Forms of Degrees 0 and 1}

We will work with finite simplicial complexes, for simplicity as well as in order to stay in the combinatorial category, although parts of Forman's theory can be used for regular cellular complexes. For relevant notions on simplicial complex the reader can consult for example [1]. For a finite simplicial complex $X$, a face $\beta$ of a simplex $\alpha \in X$ is the convex hull of a non-empty subset of the set of vertices of $\alpha$, indicated by $\beta \leq \alpha$. We write $|X|$ for the underlying space of $X$ - the union of all elements of $X$. We denote $T^{k}(X)=\{(\alpha, \beta) \in X \times X \mid \beta \leq \alpha, \operatorname{dim} \beta=\operatorname{dim} \alpha-k\}$. In particular $T^{0}(X)$ can be identified with $X$. We use the notation $v_{0} v_{1} \cdots v_{n}$ for an unoriented simplex with the set of vertices $\left\{v_{0}, v_{1}, \ldots, v_{n}\right\}$, and the notation $\left[v_{0}, v_{1}, \ldots, v_{n}\right]$ for an oriented simplex with the same set of vertices. An orientation for $X$ is a choice of orientations for all the simplexes of $X$. Let $C_{*}(X)$ be the real simplicial chain complex of $X$, so $C_{n}(X)$ is the vector space over the real numbers generated by all the oriented simplexes of $X$ of dimension 
$n$ modulo the relation that two simplexes with opposite orientations have sum equal 0 .

The following is the general definition of forms given by Forman in [5]:

Definition 1. A $d$-form is a linear map $\omega: C_{*}(X) \rightarrow C_{*}(X)$ such that for each $i$-dimensional oriented simplex $\alpha$, the chain $\omega(\alpha)$ is a linear combination of $(i-d)$-dimensional oriented simplexes which are faces of $\alpha$.

The set of $k$-forms on $X$ is denoted by $\Omega^{k}(X)$.

Let us give $X$ an orientation and give $C_{*}(X)$ an inner product such that $X$ forms an orthonormal vector basis. With this inner product we can specify a $d$-form $\omega$ by

$$
\omega(\alpha)=\sum_{\beta,(\alpha, \beta) \in T^{d}(X)}\langle\omega(\alpha), \beta\rangle \beta .
$$

There is a bijection between 0-forms on $X$ and maps from $T^{0}(X)$ to $\mathbb{R}$. Given a map $f: T^{0}(X) \rightarrow \mathbb{R}$ let $\omega: C_{*}(X) \rightarrow C_{*}(X)$ be the linear map given by for each $\alpha \in X, \omega(\alpha)=f(\alpha) \alpha$. Notice that $f$ does not depend on orientations of cells of $X$, so $\omega(-\alpha)=-\omega(\alpha)$, thus $\omega$ is well-defined as a map on $C_{*}(X)$, and is a 0 -form. In the reverse direction, given a 0 -form $\omega$, define $f(\alpha)=\langle\omega(\alpha), \alpha\rangle$. Then $f(-\alpha)=f(\alpha)$, so $f$ is a map from $X$ to $\mathbb{R}$ not depending on orientation of $X$. So a 0 -form on $X$ can be represented by a real function on $X$.

If $\alpha$ is oriented as $\left[v_{0}, v_{1}, \ldots, v_{n}\right]$ then a compatible orientation of a face of $\alpha$ is given by the orientation of $(-1)^{i}\left[v_{0}, \ldots, \hat{v}_{i}, \ldots, v_{n}\right]$. For oriented simplexes $\beta<\alpha$ and $\operatorname{dim} \beta=\operatorname{dim} \alpha-1$, the $\operatorname{sign}(\alpha, \beta)$ is 1 if the orientation of $\beta$ is compatible with the orientation of $\alpha$, and -1 if not.

The boundary of the $n$-dimensional oriented simplex $\sigma=\left[v_{0}, v_{1}, \ldots, v_{n}\right]$ is the $(n-1)$-dimensional chain $\partial_{n} \sigma=\sum_{i=0}^{n}(-1)^{i}\left[v_{0}, v_{1}, \ldots, v_{i-1}, \widehat{v}_{i}, v_{i+1}, \ldots, v_{n}\right]$, where the notation $\widehat{v}_{i}$ is traditionally used to indicate that this element is dropped. This map is extended linearly to become a map from $C_{n}(X)$ to $C_{n-1}(X)$. Using the boundary operator we can write $\operatorname{sign}(\alpha, \beta)=\langle\partial \alpha, \beta\rangle$.

For 1-forms, given a map $f: T^{1}(X) \rightarrow \mathbb{R}$ let

$$
\begin{aligned}
\omega: C_{*}(X) & \rightarrow C_{*}(X) \\
\alpha & \mapsto \sum_{\{\beta \in X \mid} \sum_{\left.(\alpha, \beta) \in T^{1}(X)\right\}} f(\alpha, \beta) \operatorname{sign}(\alpha, \beta) \beta .
\end{aligned}
$$

In other words $\langle\omega(\alpha), \beta\rangle=f(\alpha, \beta)\langle\partial \alpha, \beta\rangle$. Then $\omega(-\alpha)=-\omega(\alpha)$, and so $\omega$ is a 1 -form. The inverse correspondence is given by

$$
f(\alpha, \beta)=\langle\omega(\alpha), \beta\rangle \operatorname{sign}(\alpha, \beta)=\langle\omega(\alpha), \beta\rangle\langle\partial \alpha, \beta\rangle,
$$

which does not depend on orientations of either $\alpha$ or $\beta$. So we can represent a 1 -form on $X$ by a real function on $T^{1}(X)$.

From now on for simplicity in notation we often identify 0 and 1-forms (which often given by actions on oriented simplexes) with their representations by real functions (which act on unoriented simplexes), and use the same notations for them when there is no danger of confusion.

\section{Closedness and Exactness of Forms of Degrees 0 and 1.}

\subsection{Derivatives of 0 -forms}

The derivative of a smooth 0 -form is a measure of the rate of change of the function, therefore on simplicial complex the derivative of a function should tell how much the function changes. The following definition was given in [5]: 
Definition 2. Let $X$ be a finite simplicial complex. If $f \in \Omega^{0}(X)$, then its derivative as an element $d f \in \Omega^{1}(X)$ is defined as:

$$
\begin{aligned}
d f: T^{1}(X) & \rightarrow \mathbb{R} \\
(\alpha, \beta) & \mapsto f(\alpha)-f(\beta) .
\end{aligned}
$$

In other words $\langle d f(\alpha), \beta\rangle=(f(\alpha)-f(\beta))\langle\partial \alpha, \beta\rangle$.

As in the case of smooth forms, we say that a form is closed if its derivative is the form 0, i.e. $\omega$ is closed if $d \omega=0$. A form is exact if it is the derivative of a form, i.e. $\omega$ is exact if there is $\alpha$ such that $d \alpha=\omega$. The following analogy of a result for smooth forms was stated in [5, p. 344]:

Theorem 1. A zero form on a connected simplicial complex is closed if and only if it is constant.

To prove this result we use the following lemma:

Lemma 1. Let $X$ be a connected finite simplicial complex. If $\alpha, \beta \in X$ then there is a finite sequence $X_{0}, X_{2}, \ldots, X_{m}$ of elements of $X$ such that $X_{0}=\alpha, X_{m}=\beta$, and $X_{i} \cap X_{i+1} \neq \emptyset$ for all $0 \leq i \leq m-1$.

Proof. Let us call for the purpose of this proof a finite sequence $X_{0}, X_{2}, \ldots, X_{m} \in X$ such that $X_{i} \cap X_{i+1} \neq \emptyset$ for all $0 \leq i \leq m-1$ a path. Let

$$
S(\alpha)=\{\gamma \in X \mid \text { there is a path starting at } \alpha \text { ending at } \gamma\} .
$$

Let $T(\alpha)=\bigcup_{\gamma \in S(\alpha)} \gamma$. We prove that if $\delta \notin S(\alpha)$ then $\delta \cap T(\alpha)=\emptyset$. Indeed, otherwise there is $\gamma \in S(\alpha)$ such that $\delta \cap \gamma \neq \emptyset$. There is a chain $X_{0}, X_{2}, \ldots, X_{m} \in X$ such that $X_{0}=\alpha, X_{m}=\gamma$, and $X_{i} \cap X_{i+1} \neq \emptyset$ for all $0 \leq i \leq m-1$. Let $X_{m+1}=\delta$, then $X_{0}, X_{2}, \ldots, X_{m+1}$ is a chain starting at $\alpha$ ending at $\delta$. So $\delta \in S(\alpha)$, a contradiction.

As a consequence

$$
|X| \backslash T(\alpha)=\bigcup_{\delta \in X \backslash S(\alpha)} \delta
$$

Since each simplex is a closed set, both $T(\alpha)$ and $|X| \backslash T(\alpha)$ are disjoint closed sets. Since $X$ is connected and $T(\alpha) \neq \emptyset$, we must have $|X| \backslash T(\alpha)=\emptyset$, so $S(\alpha)=X$.

Proof of Theorem 1. Let $X$ be a connected finite simplicial complex and let $f \in \Omega^{0}(X)$.

$(\Leftarrow)$ Suppose that $f$ is constant on $X$. Then for each $(\alpha, \beta) \in T^{1}(X)$ we have $d f(\alpha, \beta)=f(\alpha)-f(\beta)=0$.

$(\Rightarrow)$ Suppose that $d f=0$.

Step 1: Let $P(n)$ be the statement that if $|X|$ is an $n$-dimensional simplex and $d f=0$ on $X$ then $f$ is constant on $X$. When $n=1$ we can write $|X|=A_{0} A_{1}$. Then $d f\left(A_{0} A_{1}, A_{0}\right)=f\left(A_{0} A_{1}\right)-f\left(A_{0}\right)=0$ and $d f\left(A_{0} A_{1}, A_{1}\right)=f\left(A_{0} A_{1}\right)-f\left(A_{1}\right)=0$, thus $f\left(A_{0} A_{1}\right)=f\left(A_{0}\right)=f\left(A_{1}\right)$. So $P(1)$ is true.

Suppose that $P(n)$ is true, we show that $P(n+1)$ is true. Write $|X|=A_{0} A_{1} \ldots A_{n+1}$. The $n$-dimensional faces of $X$ are $X_{i}=A_{0} A_{1} \ldots \widehat{A}_{i} \ldots A_{n+1}, 1 \leq i \leq n+1$. Since $d f\left(|X|, X_{i}\right)=f(|X|)-f\left(X_{i}\right)=0$ we have $f(|X|)=f\left(X_{i}\right), 1 \leq i \leq n+1$. By the induction hypothesis $f$ is constant on the set of faces of $X_{i}$, thus $f$ is constant on $X$. Thus $P(n)$ is true for all $n \geq 1$.

Step 2: We show that if $\alpha$ and $\beta$ are two elements of $X$ and $\alpha \cap \beta \neq \emptyset$ then $f(\alpha)=f(\beta)$. Indeed, let $\gamma=\alpha \cap \beta$ then $\gamma$ is a face of both $\alpha$ and $\beta$. By Step 1, we have $f(\gamma)=f(\alpha)$ and $f(\gamma)=f(\beta)$, thus $f(\alpha)=f(\beta)$.

Step 3: Let $\alpha, \beta \in X$. By Lemma 1 there is a sequence $X_{0}, X_{2}, \ldots, X_{m}$ of elements of $X$ such that $X_{0}=\alpha$, $X_{m}=\beta$, and $X_{i} \cap X_{i+1} \neq \emptyset$ for all $0 \leq i \leq m-1$. Step 2 implies that $f(\alpha)=f\left(X_{0}\right)=f\left(X_{2}\right)=\cdots=$ $f\left(X_{m}\right)=f(\beta)$. 


\subsection{Derivatives of 1 -forms}

If $\gamma<\beta<\alpha$ with $\operatorname{dim} \gamma=\operatorname{dim} \beta-1=\operatorname{dim} \alpha-2$ we define

$$
\operatorname{sign}(\alpha, \gamma, \beta)=\operatorname{sign}(\alpha, \gamma) \operatorname{sign}(\gamma, \beta)=\langle\partial \alpha, \gamma\rangle \cdot\langle\partial \gamma, \beta\rangle .
$$

Lemma 2. If $(\alpha, \beta) \in T^{2}(X)$ then there exist exactly two simplexes $\gamma_{1}, \gamma_{2} \in X$ such that $\beta<\gamma_{1}, \gamma_{2}<\alpha$, $\operatorname{dim} \gamma_{1}=\operatorname{dim} \gamma_{2}=\operatorname{dim} \beta+1$. Furthermore $\operatorname{sign}\left(\alpha, \gamma_{1}, \beta\right)=-\operatorname{sign}\left(\alpha, \gamma_{2}, \beta\right)$.

Proof. Let us write $\alpha=\left[x_{0}, x_{1}, \ldots, x_{n}\right]$, then we have $\beta=\epsilon\left[x_{0}, \ldots, \widehat{x_{i}}, \ldots, \widehat{x_{j}}, \ldots, x_{n}\right], \gamma_{1}=\epsilon_{1}\left[x_{0}, \ldots, \widehat{x_{i}}, \ldots, x_{n}\right]$, and $\gamma_{2}=\epsilon_{2}\left[x_{0}, \ldots, \widehat{x_{j}}, \ldots, x_{n}\right]$, where $\epsilon, \epsilon_{1}, \epsilon_{2}$ are \pm 1 , and $i<j$. We find $\operatorname{sign}\left(\alpha, \gamma_{1}\right)=\epsilon_{1}(-1)^{i}, \operatorname{sign}\left(\alpha, \gamma_{2}\right)=$ $\epsilon_{2}(-1)^{j}, \operatorname{sign}\left(\gamma_{1}, \beta\right)=\epsilon \epsilon_{1}(-1)^{j-1}, \operatorname{sign}\left(\gamma_{2}, \beta\right)=\epsilon \epsilon_{2}(-1)^{i}$. Thus $\operatorname{sign}\left(\alpha, \gamma_{1}, \beta\right)=\epsilon \epsilon_{1}^{2}(-1)^{i+j-1}$ while $\operatorname{sign}\left(\alpha, \gamma_{2}, \beta\right)=\epsilon \epsilon_{2}^{2}(-1)^{i+j}=-\operatorname{sign}\left(\alpha, \gamma_{1}, \beta\right)$.

Definition 3 ([5, p. 345]). For a 1-form $\omega \in \Omega^{1}(X)$ we define its derivative $d \omega \in \Omega^{2}(X)$ as the 2 -form defined by for $(\alpha, \beta) \in T^{2}(X)$,

$$
\langle(d \omega)(\alpha), \beta\rangle=\operatorname{sign}\left(\alpha, \gamma_{1}, \beta\right)\left(\omega\left(\alpha, \gamma_{1}\right)+\omega\left(\gamma_{1}, \beta\right)\right)+\operatorname{sign}\left(\alpha, \gamma_{2}, \beta\right)\left(\omega\left(\alpha, \gamma_{2}\right)+\omega\left(\gamma_{2}, \beta\right)\right),
$$

where $\alpha>\gamma_{1}, \gamma_{2}>\beta$.

We can rewrite the formula of $d \omega$ without using representations of forms by real functions as

$$
\begin{aligned}
\langle(d \omega)(\alpha), \beta\rangle= & \left\langle\partial \alpha, \gamma_{1}\right\rangle\left\langle\partial \gamma_{1}, \beta\right\rangle\left(\left\langle\omega(\alpha), \gamma_{1}\right\rangle\left\langle\partial \alpha, \gamma_{1}\right\rangle+\left\langle\omega\left(\gamma_{1}\right), \beta\right\rangle\left\langle\partial \gamma_{1}, \beta\right\rangle\right)+ \\
& +\left\langle\partial \alpha, \gamma_{2}\right\rangle\left\langle\partial \gamma_{2}, \beta\right\rangle\left(\left\langle\omega(\alpha), \gamma_{2}\right\rangle\left\langle\partial \alpha, \gamma_{2}\right\rangle+\left\langle\omega\left(\gamma_{2}\right), \beta\right\rangle\left\langle\partial \gamma_{2}, \beta\right\rangle\right) \\
= & \left\langle\omega(\alpha), \gamma_{1}\right\rangle\left\langle\partial \gamma_{1}, \beta\right\rangle+\left\langle\omega\left(\gamma_{1}\right), \beta\right\rangle\left\langle\partial \alpha, \gamma_{1}\right\rangle+ \\
& +\left\langle\omega(\alpha), \gamma_{2}\right\rangle\left\langle\partial \gamma_{2}, \beta\right\rangle+\left\langle\omega\left(\gamma_{2}\right), \beta\right\rangle\left\langle\partial \alpha, \gamma_{2}\right\rangle .
\end{aligned}
$$

We recognize that $\langle(d \omega)(-\alpha), \beta\rangle=-\langle(d \omega)(\alpha), \beta\rangle$, thus $d \omega$ is indeed a map on $C_{*}(X)$.

Immediately from definition 3 and Lemma 2 we have the following result, [5, p. 345]:

Proposition 1. A 1-form $\omega$ is closed if and only if

$$
\omega\left(\alpha, \gamma_{1}\right)+\omega\left(\gamma_{1}, \beta\right)=\omega\left(\alpha, \gamma_{2}\right)+\omega\left(\gamma_{2}, \beta\right)
$$

for all $\beta<\gamma_{1}, \gamma_{2}<\alpha$.

As a corollary:

Proposition 2. For 1-forms, being exact implies being closed.

Proof. Suppose that $\omega=d f$. Then for all $(\alpha, \beta) \in T^{2}(X)$, by Lemma 2

$$
\begin{aligned}
\langle(d \omega)(\alpha), \beta\rangle= & \operatorname{sign}\left(\alpha, \gamma_{1}, \beta\right)\left(\omega\left(\alpha, \gamma_{1}\right)+\omega\left(\gamma_{1}, \beta\right)\right)+\operatorname{sign}\left(\alpha, \gamma_{2}, \beta\right)\left(\omega\left(\alpha, \gamma_{2}\right)+\omega\left(\gamma_{2}, \beta\right)\right) \\
= & \operatorname{sign}\left(\alpha, \gamma_{1}, \beta\right)\left(f(\alpha)-f\left(\gamma_{1}\right)+f\left(\gamma_{1}\right)-f(\beta)\right)+ \\
& +\operatorname{sign}\left(\alpha, \gamma_{2}, \beta\right)\left(f(\alpha)-f\left(\gamma_{2}\right)+f\left(\gamma_{2}\right)-f(\beta)\right) \\
= & \left(\operatorname{sign}\left(\alpha, \gamma_{1}, \beta\right)+\operatorname{sign}\left(\alpha, \gamma_{2}, \beta\right)\right)(f(\alpha)-f(\beta))=0 .
\end{aligned}
$$

\subsection{A closed but not exact 1-form.}

For smooth forms, there is an angular form defined on the plane minus a point, which is a closed but not exact 1 -form. Its existence is due to the fact that its domain is not simply connected. For combinatorial forms we would like to see a similar example. 


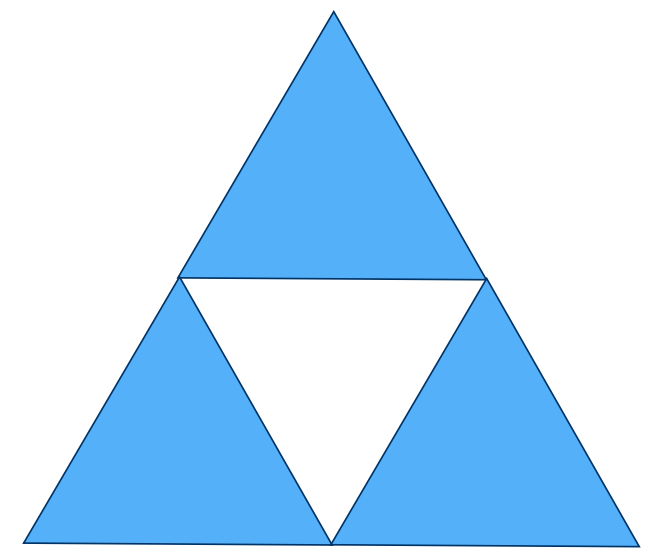

Figure 1:

Let $X$ be the simplicial complex formed by the triangles $A_{1} A_{2} A_{3}, A_{2} A_{4} A_{5}, A_{3} A_{5} A_{6}$ in Figure 1 . The underlying space is not simply-connected.

To give a closed 1-form $\omega$ on $X$, on the triangle $A_{1} A_{2} A_{3}$ we assign arbitrary values for $\omega\left(A_{1} A_{2} A_{3}, A_{1} A_{2}\right)$, $\omega\left(A_{1} A_{2} A_{3}, A_{2} A_{3}\right), \omega\left(A_{1} A_{2} A_{3}, A_{3} A_{1}\right), \omega\left(A_{1} A_{2}, A_{1}\right), \omega\left(A_{2} A_{3}, A_{2}\right), \omega\left(A_{3} A_{1}, A_{3}\right)$. By the condition for closed forms:

$$
\omega\left(A_{1} A_{2} A_{3}, A_{1} A_{2}\right)+\omega\left(A_{1} A_{2}, A_{1}\right)=\omega\left(A_{1} A_{2} A_{3}, A_{3} A_{1}\right)+\omega\left(A_{3} A_{1}, A_{1}\right),
$$

thus $\omega\left(A_{3} A_{1}, A_{1}\right)$ is accordingly determined from the initial assignment. Similarly $\omega\left(A_{1} A_{2}, A_{2}\right)$ and $\omega\left(A_{2} A_{3}, A_{3}\right)$ are accordingly determined. Assignments on the triangles $A_{2} A_{4} A_{5}$ and $A_{3} A_{5} A_{6}$ can be done similarly and are independent from the assignment on the triangle $A_{1} A_{2} A_{3}$. This may lead to a conflict at common vertices of the three triangles. Indeed, now we give a concrete example.

Define a 1 -form $\omega$ as:

$\omega\left(A_{1} A_{2} A_{3}, A_{1} A_{2}\right)=1, \omega\left(A_{1} A_{2} A_{3}, A_{3} A_{1}\right)=2, \omega\left(A_{1} A_{2} A_{3}, A_{2} A_{3}\right)=3, \omega\left(A_{1} A_{2}, A_{1}\right)=4, \omega\left(A_{1} A_{2}, A_{2}\right)=$ $5, \omega\left(A_{1} A_{3}, A_{1}\right)=3, \omega\left(A_{1} A_{3}, A_{3}\right)=6, \omega\left(A_{2} A_{3}, A_{2}\right)=3, \omega\left(A_{2} A_{3}, A_{3}\right)=5, \omega\left(A_{2} A_{4} A_{5}, A_{2} A_{4}\right)=7$, $\omega\left(A_{2} A_{4} A_{5}, A_{2} A_{5}\right)=8, \omega\left(A_{2} A_{4} A_{5}, A_{4} A_{5}\right)=9, \omega\left(A_{2} A_{4}, A_{2}\right)=10, \omega\left(A_{2} A_{4}, A_{4}\right)=11, \omega\left(A_{2} A_{5}, A_{2}\right)=9$, $\omega\left(A_{2} A_{5}, A_{5}\right)=12, \omega\left(A_{4} A_{5}, A_{4}\right)=9, \omega\left(A_{4} A_{5}, A_{5}\right)=11, \omega\left(A_{3} A_{5} A_{6}, A_{3} A_{5}\right)=13, \omega\left(A_{3} A_{5} A_{6}, A_{3} A_{6}\right)=14$, $\omega\left(A_{3} A_{5} A_{6}, A_{5} A_{6}\right)=15, \omega\left(A_{3} A_{5}, A_{3}\right)=0, \omega\left(A_{3} A_{5}, A_{5}\right)=5, \omega\left(A_{3} A_{6}, A_{3}\right)=-1, \omega\left(A_{3} A_{6}, A_{6}\right)=10$, $\omega\left(A_{5} A_{6}, A_{5}\right)=3, \omega\left(A_{5} A_{6}, A_{6}\right)=9$.

Proposition 3. The form $\omega$ is closed.

Proof. We check all possible relations from Proposition 1 , by direct computation:

$$
\begin{aligned}
& \omega\left(A_{1} A_{2} A_{3}, A_{1} A_{2}\right)+\omega\left(A_{1} A_{2}, A_{1}\right)=\omega\left(A_{1} A_{2} A_{3}, A_{1} A_{3}\right)+\omega\left(A_{1} A_{3}, A_{1}\right)=5 \\
& \omega\left(A_{1} A_{2} A_{3}, A_{1} A_{2}\right)+\omega\left(A_{1} A_{2}, A_{2}\right)=\omega\left(A_{1} A_{2} A_{3}, A_{2} A_{3}\right)+\omega\left(A_{2} A_{3}, A_{2}\right)=6 \\
& \omega\left(A_{1} A_{2} A_{3}, A_{1} A_{3}\right)+\omega\left(A_{1} A_{3}, A_{3}\right)=\omega\left(A_{1} A_{2} A_{3}, A_{2} A_{3}\right)+\omega\left(A_{2} A_{3}, A_{3}\right)=8 \\
& \omega\left(A_{2} A_{4} A_{5}, A_{2} A_{4}\right)+\omega\left(A_{2} A_{4}, A_{2}\right)=\omega\left(A_{2} A_{4} A_{5}, A_{2} A_{5}\right)+\omega\left(A_{2} A_{5}, A_{2}\right)=17 \\
& \omega\left(A_{2} A_{4} A_{5}, A_{2} A_{4}\right)+\omega\left(A_{2} A_{4}, A_{4}\right)=\omega\left(A_{2} A_{4} A_{5}, A_{4} A_{5}\right)+\omega\left(A_{4} A_{5}, A_{4}\right)=18 \\
& \omega\left(A_{2} A_{4} A_{5}, A_{2} A_{5}\right)+\omega\left(A_{2} A_{5}, A_{5}\right)=\omega\left(A_{2} A_{4} A_{5}, A_{4} A_{5}\right)+\omega\left(A_{4} A_{5}, A_{5}\right)=20 \\
& \omega\left(A_{3} A_{5} A_{6}, A_{3} A_{5}\right)+\omega\left(A_{3} A_{5}, A_{3}\right)=\omega\left(A_{3} A_{5} A_{6}, A_{3} A_{6}\right)+\omega\left(A_{3} A_{6}, A_{3}\right)=13 \\
& \omega\left(A_{3} A_{5} A_{6}, A_{3} A_{5}\right)+\omega\left(A_{3} A_{5}, A_{5}\right)=\omega\left(A_{3} A_{5} A_{6}, A_{5} A_{6}\right)+\omega\left(A_{5} A_{6}, A_{5}\right)=18 \\
& \omega\left(A_{3} A_{5} A_{6}, A_{3} A_{6}\right)+\omega\left(A_{3} A_{6}, A_{6}\right)=\omega\left(A_{3} A_{5} A_{6}, A_{5} A_{6}\right)+\omega\left(A_{5} A_{6}, A_{6}\right)=24 .
\end{aligned}
$$


Proposition 4. The form $\omega$ is not exact.

Proof. Suppose that $\omega$ is exact then there is a function $f$ defined on $X$ satisfying $\omega=d f$. Let $a=$ $f\left(A_{1} A_{2} A_{3}\right)$, we get:

$$
\begin{aligned}
f\left(A_{1} A_{2}\right) & =f\left(A_{1} A_{2} A_{3}\right)-\omega\left(A_{1} A_{2} A_{3}, A_{1} A_{2}\right)=a-1, \\
f\left(A_{2}\right) & =f\left(A_{1} A_{2}\right)-\omega\left(A_{1} A_{2}, A_{2}\right)=a-6, \\
f\left(A_{1} A_{3}\right) & =f\left(A_{1} A_{2} A_{3}\right)-\omega\left(A_{1} A_{2} A_{3}, A_{1} A_{3}\right)=a-2, \\
f\left(A_{3}\right) & =f\left(A_{1} A_{3}\right)-\omega\left(A_{1} A_{3}, A_{3}\right)=a-8, \\
f\left(A_{2} A_{5}\right) & =f\left(A_{2}\right)+\omega\left(A_{2} A_{5}, A_{2}\right)=a+3, \\
f\left(A_{5}\right) & =f\left(A_{2} A_{5}\right)-\omega\left(A_{2} A_{5}, A_{5}\right)=a-9, \\
f\left(A_{3} A_{5}\right) & =f\left(A_{5}\right)+\omega\left(A_{3} A_{5}, A_{5}\right)=a-4, \\
f\left(A_{3}\right) & =f\left(A_{3} A_{5}\right)-\omega\left(A_{3} A_{5}, A_{3}\right)=a-4 .
\end{aligned}
$$

At this point we observe that $f$ receives two different values at $A_{3}$, a contradiction. Thus $\omega$ is not exact.

\subsection{Closed 1-forms which are exact}

In this subsection we prove a positive result on cases where a closed 1-form is exact, without using Forman's de Rham-type theorem [5, p. 341].

Theorem 2. Any closed one-form on a simplex is exact.

Proof. Let $P(n)$ be the following statement: Given an $n$-dimensional simplicial complex $X$ such that $|X|$ is a simplex, a closed 1-form $\omega$ on $X$, and a real number $c$, there is a unique 0 -form $f$ on $X$ such that $d f=\omega$ and $f(|X|)=c$. We will use induction to prove that $P(n)$ is true for all $n \geq 1$.

The case $n=1$ is trivial, and the case $n=2$ has been proved in Proposition 1 above.

Supposing $P(n)$ is true, we prove that $P(n+1)$ is true. Let $f(|X|)=c$.

For any $n$-dimensional face $\sigma$ of $|X|$, define $f(\sigma)=f_{\sigma}(\sigma)=f(|X|)-\omega(|X|, \sigma)$. By the induction hypothesis, $f_{\sigma}$ is then defined uniquely on the simplicial complex $X_{\sigma}=\{\eta \mid \eta \leq \sigma\}$ such that $d f_{\sigma}=\omega$ on $X_{\sigma}$. It remains to be proved that $f$ is well-defined, that is, if $\eta$ is a common face of $\sigma$ and $\sigma^{\prime}$ where $\sigma \neq \sigma^{\prime}$ and $\operatorname{dim} \sigma=\operatorname{dim} \sigma^{\prime}=n$ then $f_{\sigma}(\eta)=f_{\sigma^{\prime}}(\eta)$.

Since $|X|$ is a simplex, $\alpha=\sigma \cap \sigma^{\prime}$ is a simplex of dimension $(n-1)$. By closedness of $\omega$ :

$$
\omega(|X|, \sigma)+\omega(\sigma, \alpha)=\omega\left(|X|, \sigma^{\prime}\right)+\omega\left(\sigma^{\prime}, \alpha\right) .
$$

This implies

$$
f(|X|)-f_{\sigma}(\sigma)+f_{\sigma}(\sigma)-f_{\sigma}(\alpha)=f(|X|)-f_{\sigma^{\prime}}\left(\sigma^{\prime}\right)+f_{\sigma^{\prime}}\left(\sigma^{\prime}\right)-f_{\sigma^{\prime}}(\alpha),
$$

that is $f_{\sigma}(\alpha)=f_{\sigma^{\prime}}(\alpha)$. Define $f(\alpha)=f_{\sigma}(\alpha)=f_{\sigma^{\prime}}(\alpha)$. Any common face $\eta$ of $\sigma$ and $\sigma^{\prime}$ of dimension less than $(n-1)$ is a face of $\alpha$, and since $f_{\sigma}(\alpha)=f_{\sigma^{\prime}}(\alpha)$, by uniqueness from the induction hypothesis applied to $\alpha$, we must have $f_{\sigma}(\eta)=f_{\sigma^{\prime}}(\eta)$, and we define $f(\eta)$ by this number. The proof is completed.

In a simpler case as on a triangle, we can give a straight forward proof and construct the anti-derivative function, by "integrating", as Forman put it, in a similar fashion to the smooth case:

Example 1. Any closed 1-form on a 2-dimensional simplex is exact.

Let $X$ be the simplicial complex formed by the triangle $A B C$ in Figure 2 , and let $\omega$ be a closed 1-form on $X$.

Let $f(A B C)$ be any real number. Define

$$
\begin{aligned}
f(A B) & =-\omega(A B C, A B)+f(A B C), f(A C)=-\omega(A B C, A C)+f(A B C) \\
f(B C) & =-\omega(A B C, B C)+f(A B C), f(A)=-\omega(A B C, A B)-\omega(A B, A)+f(A B C) \\
f(B) & =-\omega(A B C, A B)-\omega(A B, B)+f(A B C), f(C)=-\omega(A B C, A C)-\omega(A C, C)+f(A B C) .
\end{aligned}
$$




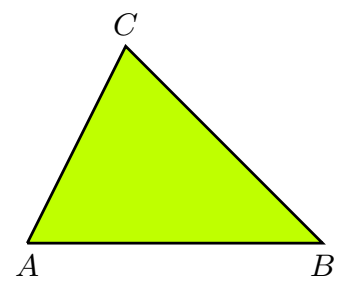

Figure 2:

Then

$$
\begin{aligned}
\omega(A B C, A B) & =f(A B C)-f(A B), \omega(A B C, B C)=f(A B C)-f(B C), \\
\omega(A B C, C A) & =f(A B C)-f(C A), \omega(A B, A)=f(A B)-f(A), \\
\omega(A B, B) & =f(A B)-f(B), \omega(A C, C)=f(A C)-f(C) .
\end{aligned}
$$

Using the closedness of $\omega$ we have

$$
\begin{aligned}
& \omega(C A, A)=(\omega(A B C, A B)+\omega(A B, A))-\omega(A B C, A C)=f(C A)-f(A), \\
& \omega(B C, B)=(\omega(A B C, A B)+\omega(A B, B))-\omega(A B C, B C)=f(B C)-f(B), \\
& \omega(B C, C)=(\omega(A B C, A C)+\omega(A C, C))-\omega(A B C, B C)=f(B C)-f(C) .
\end{aligned}
$$

Thus $d f=\omega$.

\section{Products and Pullbacks of Forms}

\subsection{Products of forms}

Forman [5, p. 341] suggested that product of forms is by composition of maps: If $\omega_{1} \in \Omega^{d_{1}}(X)$ and $\omega_{2} \in \Omega^{d_{2}}(X)$ then

$$
\omega_{1} \wedge \omega_{2}=\omega_{1} \circ \omega_{2} \in \Omega^{d_{1}+d_{2}}(X) .
$$

In other words, for $\alpha \in C_{*}(X)$ we have

$$
\left(\omega_{1} \wedge \omega_{2}\right)(\alpha)=\omega_{1}\left(\omega_{2}(\alpha)\right) .
$$

In this definition the product has certain properties analogous to the smooth counterpart, such as associativity, from the associativity of compositions of maps: for any forms $\omega_{1}, \omega_{2}, \omega_{3}$ we have

$$
\omega_{1} \wedge\left(\omega_{2} \wedge \omega_{3}\right)=\left(\omega_{1} \wedge \omega_{2}\right) \wedge \omega_{3} .
$$

Recall that the product of smooth differential forms is anti-commutative, that is, if $\omega_{1}$ is a $d_{1}$-form and $\omega_{2}$ is a $d_{2}$-form then

$$
\omega_{1} \wedge \omega_{2}=(-1)^{d_{1}+d_{2}} \omega_{2} \wedge \omega_{1} .
$$

Since product for combinatorial form is composition of linear maps, in other words, product of matrices, it is not likely that commutativity or anti-commutativity hold. Indeed we can give an example of 1-forms whose products are neither commutative nor anti-commutative.

Let $X$ be the simplicial complex formed by the triangle $A B C$ in Figure 2 . Consider the 1-forms $\omega_{1}$ and $\omega_{2}$ defined on $X$ as:

$$
\begin{gathered}
\omega_{1}([A, B])=2 A-B, \omega_{1}([B, C])=-3 B+5 C, \omega_{1}([C, A])=A+4 C, \\
\omega_{1}([A, B, C])=-[B, C]+2[C, A]+[A, B],
\end{gathered}
$$


and

$$
\begin{gathered}
\omega_{2}([A, B])=-A+3 B, \omega_{2}([B, C])=4 B-2 C, \omega_{2}([A, C])=3 A+C, \\
\omega_{2}([A, B, C])=[B, C]-3[C, A]+2[A, B] .
\end{gathered}
$$

Then

$$
\left(\omega_{1} \wedge \omega_{2}\right)([A, B, C])=\omega_{1}\left(\omega_{2}([A, B, C])\right)=\omega_{1}([B, C]-3[C, A]+2[A, B])=A-5 B-7 C,
$$

while

$$
\left(\omega_{2} \wedge \omega_{1}\right)([A, B, C])=\omega_{2}\left(\omega_{1}([A, B, C])\right)=\omega_{2}(-[B, C]+2[C, A]+[A, B])=5 A-B+4 C .
$$

So we obtain:

Proposition 5. There exist 1-forms $\omega_{1}$ and $\omega_{2}$ on $[A, B, C]$ such that $\left(\omega_{1} \wedge \omega_{2}\right)([A, B, C]) \neq\left(\omega_{2} \wedge\right.$ $\left.\omega_{1}\right)([A, B, C])$ and $\left(\omega_{1} \wedge \omega_{2}\right)([A, B, C]) \neq-\left(\omega_{2} \wedge \omega_{1}\right)([A, B, C])$.

Thus the proposed product of 1 -forms is neither commutative nor anti-commutative, differing significantly to the smooth counterpart.

\subsection{Pullbacks of forms}

Pulling back of forms is an important operation for smooth forms, bringing forms on one space to forms on another space, as a generalization of change of variables in differentiation. Forman has not discussed pullbacks of combinatorial forms. Here we propose a definition for pullbacks of combinatorial forms, then we investigate several initial properties.

Definition 4. Given two oriented simplicial complexes $X, Y$ and let $\omega$ be a form of degree $d$ on $X$. Let $f: Y \rightarrow X$ be such that

$$
\forall \alpha, \beta \in Y,(\beta<\alpha, \operatorname{dim} \beta=\operatorname{dim} \alpha-d) \Rightarrow(f(\beta)<f(\alpha), \operatorname{dim} f(\beta)=\operatorname{dim} f(\alpha)-d),
$$

equivalently, using different notations:

$$
\forall(\alpha, \beta) \in T^{d}(Y),(f(\alpha), f(\beta)) \in T^{d}(X)
$$

The pullback of $\omega$ by $f$ is a form of degree $d$ on $Y$, denoted by $f^{*} \omega$, and is defined by

$$
\left(f^{*} \omega\right)(\alpha)=\sum_{\beta,(\alpha, \beta) \in T^{d}(Y)}\langle\omega(f(\alpha)), f(\beta)\rangle \beta .
$$

Thus essentially, and precisely in the cases of 0 -forms and 1-forms, the value of the pullback $f^{*} \omega$ at the pair $(\alpha, \beta)$ is the value of $\omega$ at the pair $(f(\alpha), f(\beta))$.

We shall show that this pullback is linear and is natural:

Theorem 3. Let $f: Y \rightarrow X$ and $g: Z \rightarrow Y$ be maps satisfying the assumption (1) and let $\omega$ be a form of degree $d$ on $X$. Then:

1. $f^{*}(\omega+\varphi)=f^{*} \omega+f^{*} \varphi, \forall \omega, \varphi \in \Omega^{d}(X)$.

2. $g^{*}\left(f^{*} \omega\right)=(f \circ g)^{*} \omega, \forall \omega \in \Omega^{d}(X)$. 
Proof. (a) For $\alpha \in Y$, we have

$$
\begin{aligned}
f^{*}(\omega+\varphi)(\alpha) & =\sum_{\beta,(\alpha, \beta) \in T^{d}(Y)}\langle(\omega+\varphi)(f(\alpha)), f(\beta)\rangle \beta \\
& =\sum_{\beta,(\alpha, \beta) \in T^{d}(Y)}\langle\omega(f(\alpha))+\varphi(f(\alpha)), f(\beta)\rangle \beta \\
& =\sum_{\beta,(\alpha, \beta) \in T^{d}(Y)}\langle\omega(f(\alpha)), f(\beta)\rangle \beta+\sum_{\beta,(\alpha, \beta) \in T^{d}(Y)}\langle\varphi(f(\alpha)), f(\beta)\rangle \beta \\
& =f^{*} \omega(\alpha)+f^{*} \varphi(\alpha) .
\end{aligned}
$$

(b) For $\alpha \in Z$, we have

$$
\begin{aligned}
g^{*}\left(f^{*} \omega\right)(\alpha) & =\sum_{\beta,(\alpha, \beta) \in T^{d}(Z)}\left\langle f^{*} \omega(g(\alpha)), g(\beta)\right\rangle \beta \\
& =\sum_{\beta,(\alpha, \beta) \in T^{d}(Z)}\left\langle\sum_{\gamma,(g(\alpha), \gamma) \in T^{d}(Y)}\langle\omega(f(g(\alpha))), f(\gamma)\rangle \gamma, g(\beta)\right\rangle \beta \\
& =\sum_{\beta,(\alpha, \beta) \in T^{d}(Z)}\left(\sum_{\gamma,(g(\alpha), \gamma) \in T^{d}(Y)}\langle\omega(f(g(\alpha))), f(\gamma)\rangle\langle\gamma, g(\beta)\rangle\right) \beta
\end{aligned}
$$

Noticing that the inner product on $C_{*}(Y)$ receives $Y$ as an orthonormal basis, we have

$$
\langle\gamma, g(\beta)\rangle= \begin{cases}1, & \gamma=g(\beta) \\ 0, & \gamma \neq g(\beta)\end{cases}
$$

Also we notice that $(g(\alpha), g(\beta)) \in T^{d}(Y)$ by assumption on $g$. Thus

$$
g^{*}\left(f^{*} \omega\right)(\alpha)=\sum_{\beta,(\alpha, \beta) \in T^{d}(Z)}\langle\omega(f(g(\alpha))), f(g(\beta))\rangle \beta=(f \circ g)^{*} \omega(\alpha) .
$$

\section{Conclusions}

We have studied several properties regarding closed and exact 0 -forms and 1-forms, and we have proposed a linear and natural pullback operation. In view of the above results, further investigations to combinatorial forms and operations on them and comparisons of various possible approaches could be fruitful topics for future studies.

\section{References}

[1] Amstrong, M. A., 1983, Basic Topology, Springer-Verlag.

[2] Bredon, G. E., 1993, Topology and Geometry, Springer.

[3] Desbrun, M., Hirani, A. N., Leok, M. and Marsden, J. E., 2005, Discrete exterior calculus, arXiv math/0508341.

[4] Forman, R., 2002, A user's guide to discrete Morse theory, Sém. Lothar. Combin., 48, 1 - 35.

[5] Forman, R., 2002, Combinatorial Novikov-Morse theory, International Journal of Mathematics, 13(4), $333-368$. 
Derivatives, Products and Pullbacks in Forman's Combinatorial Differential Forms

[6] Forman, R., 2005, Some applications of combinatorial differential topology, Graphs and Patterns in Mathematics and Theoretical Physics, of Proc. Sympos. Pure Math., 73, 281 - 313.

[7] Guillemin, V. and Pollack, A., 1974, Differential topology, Prentice-Hall.

[8] Elizabeth, L. M. and Hydon, P. E., 2008, Difference forms, Foundations of Computational Mathematics, 8(4), 427-467.

[9] Munkres, J. R., 1991, Analysis on manifolds, Addison Wesley. 